\title{
Resonance parameter covariance representation: file32 versus file33
}

\author{
Luiz Leal ${ }^{*}$ \\ Institut de Radioprotection et de Sûreté Nucléaire (IRSN), PSN-EXP, SNC, 92260 Fontenay-aux-Roses, France
}

Received: 1 December 2017 / Received in final form: 25 January 2018 / Accepted: 28 May 2018

\begin{abstract}
Sensitivity and uncertainty analysis in error propagation studies are carried out based on nuclear data uncertainty information available in the basic nuclear data libraries such as ENDF, JEFF, JENDL and others. The uncertainty files (covariance matrices) are generally obtained from analysis of experimental data. In the resonance region, the computer code SAMMY is used for analyses of experimental data and generation of resonance parameters. In addition to resonance parameters evaluation, SAMMY also generates resonance parameter covariance matrices (RPCM). The intent of this paper is to discuss the use of the RPCM in contrast to the use of a cross section covariance matrix (CSCM) representation. For so, the resonance evaluation for ${ }^{48} \mathrm{Ti}$ in the resonance range from $10^{-5} \mathrm{eV}$ to $400 \mathrm{keV}$ is used. The RPCM is translated into two distinct CSCM by using a coarser and a finer group structures. The results are presented and discussions of the feasibility of using these covariance representations are depicted.
\end{abstract}

\section{Introduction}

The objective of a resolved resonance parameters evaluation is to generate parameters which combined with a resonance formalism, the R-matrix methodology for instance, lead to a reasonable representation of the experimental data such as the total, capture, fission cross sections, and etc. Along with the resonance parameters $(\mathrm{RP})$, resonance parameter covariance matrices (RPCM) are also obtained. For practical applications the RP parameters are converted to the evaluated nuclear data file format (ENDF) in the so-called FILE2 representations whereas the RPCM is converted to the ENDF FILE32 representation. While the FILE32 representation is preferred, however situations may arise whereby the use of the cross section covariance matrix (CSCM) is recommended which consists of translating FILE32 into FILE33. For instance, if the RP evaluation results in a large set of RP a drawback may be connected to computer's disk storage of the RPCM. Nevertheless, the process of converting to FILE33 is carried out based on two assumptions: a) the CSCM are provided in the energymultigroup form; b) a weighting spectrum is needed for the cross-section averaging. The CSCM provides the variances of the cross section within a specified energy region and also the correlation between cross sections of nearby energy regions. The objective of this paper is to examine the use of the CSSM representation in lieu of the RPCM. The studies are performed by way of using the RPCM

\footnotetext{
* e-mail: luiz.leal@irsn.fr
}

generated on the basis of a resolved resonance evaluation carried out for ${ }^{48} \mathrm{Ti}$. A brief description of the resonance of ${ }^{48} \mathrm{Ti}$ will be introduced prior to the discussion on the use of the CSCM. The paper will examine whether the FILE32 and FILE33 conversion grants an alternative leading to comparable calculated group covariance. The adequacy of energy group choice for the CSCM representation in the nuclear data library will be investigated.

\section{$2{ }^{48} \mathrm{Ti}$ resolved resonance evaluation}

In natural titanium the most abundant isotope is ${ }^{48} \mathrm{Ti}$ which has an abundance of $73.72 \%$ in weight. Additionally, ${ }^{48} \mathrm{Ti}$ has the largest thermal capture cross section among the natural titanium isotopes. In response to needs for criticality safety applications, revision and evaluation of nuclear systems in which titanium is present were requested. It was identified that existing titanium evaluation should be revised and a new evaluation should be put in place. Hence, an R-matrix resonance evaluation based on the code SAMMY [1] was performed [2] for ${ }^{48} \mathrm{Ti}$ in the energy range from thermal to $400 \mathrm{keV}$. In addition, the uncertainty in the cross sections were derived from resonance parameter covariance obtained in the evaluation. Since no reaction other than total, scattering and capture exists below $400 \mathrm{keV}$, the resonance parameters are uniquely determined in the evaluation by the energy of the resonance, the neutron width, the capture width, and the resonance spin. Correspondingly to these parameters are the associated variances as well as their correlations. For instance, for two resonance parameters $p_{j}$ and $p_{k}$, which can 


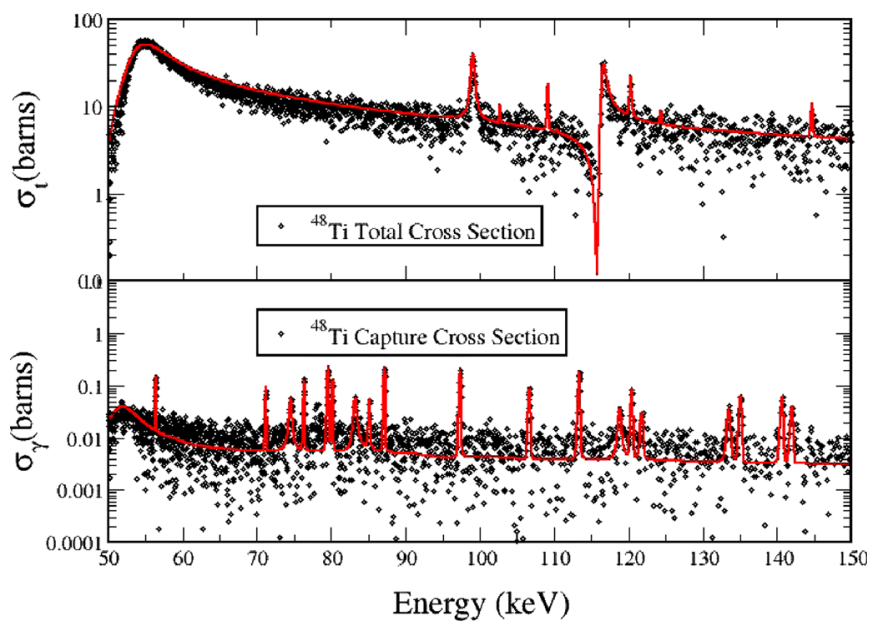

Fig. 1. SAMMY fit of the experimental differential total and capture cross sections in the energy range $50 \mathrm{keV}-150 \mathrm{keV}$ [3].

be energy or widths, their correlations leading to the $\mathrm{RPCM}$ is referred to as $\left\langle\delta p_{j} \delta p_{k}\right\rangle$. More on the practical application of the RPCM will be seen in section 3. As an example of the use the resonance parameter to reproduce the experimental cross section, the fitting of the differential total and capture cross sections in the energy range of $50-150 \mathrm{keV}$ is displayed in Figure 1.

The dots are the experimental data whereas the solid line going through the data represents the evaluation. The fitting of the experimental data was done using the ReichMoore formalism included in the code SAMMY in conjunction with the Bayes' methodology. In addition to the cross-section fitting the uncertainty in the nuclear data can also be extracted from the evaluation process in which resonance parameter covariance are obtained. The data uncertainties are statistical and systematic. The latter is the predominant data uncertainty. The uncertainties information on the experimental data used in the resonance evaluation are as follow: The observed total and capture cross section systematic uncertainties are in between 1-4\% and $2-10 \%$, respectively. These uncertainties are reflected in the final RPCM and consequently in the cross sections. Figure 2 illustrates the uncertainties and covariance for the ${ }^{48} \mathrm{Ti}$ evaluated capture cross section in the energy region from $10^{-5} \mathrm{eV}$ to $20 \mathrm{MeV}$. The covariance information is used in the analysis and design of nuclear systems. The SAMMY generated ${ }^{48} \mathrm{Ti}$ RPCM were converted into the ENDF format [4] on the basis of the LCOMP $=1$ option. This option requires that the entire covariance data are given for the full RPCM covering the resolved resonance region. Thermal values and capture resonance integral for the ${ }^{48} \mathrm{Ti}$ evaluation are compared with that included in the ENDF/B-VII.0. The results shown in Table 1 include the uncertainty in the cross sections that were calculated from the covariance derived from the resonance evaluation as indicated in the example shown in Figure 2. It should be pointed out that the most recent releases of ENDF (ENDF/ B-VIII.beta5) and JEFF (JEFF3.3T4) include the new ${ }^{48} \mathrm{Ti}$ resonance parameter evaluation.

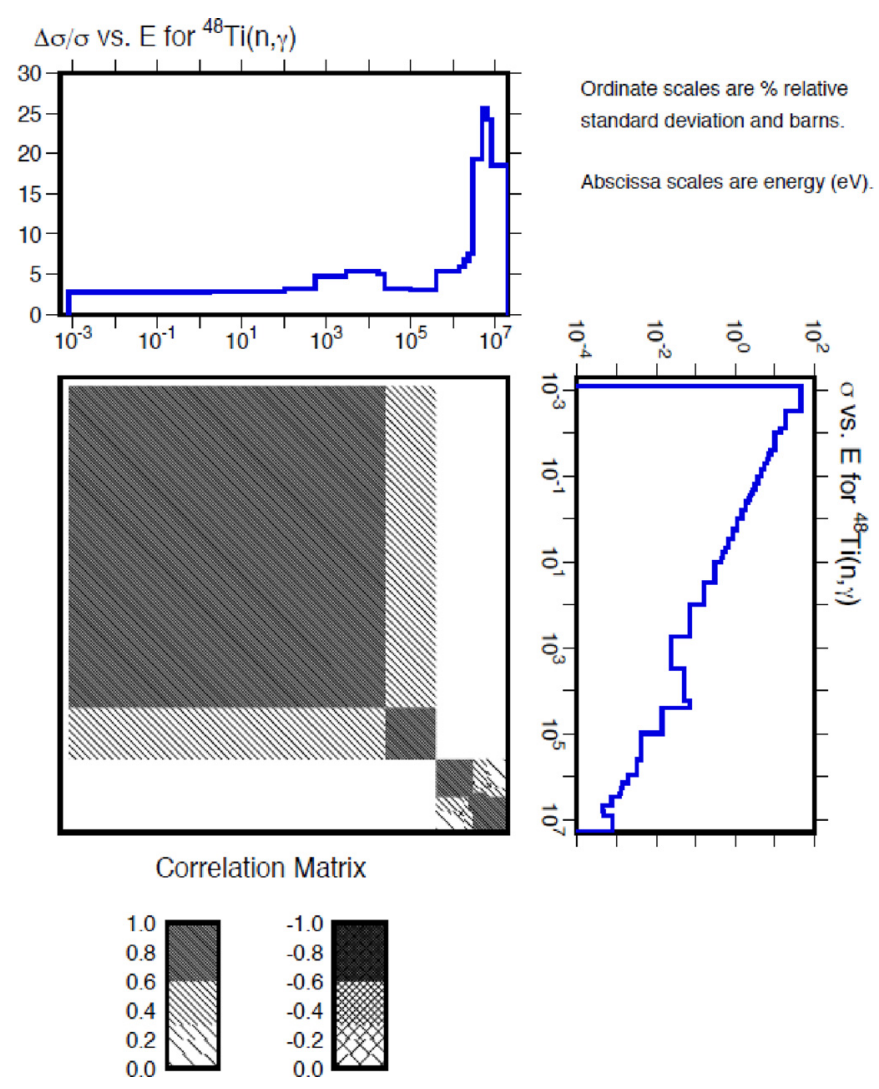

Fig. 2. Uncertainty and correlation in the Ti capture cross section using 44-energy groups.

\section{Covariance generation}

\subsection{File32 into File33}

For an energy-group with energy boundary $E_{g}$ and $E_{g+1}$ the group cross section covariance for a reaction $x$ as a function of the resonance parameter covariance is given as

$$
\left\langle\delta \bar{\sigma}_{x g} \delta \bar{\sigma}_{x g^{\prime}}\right\rangle=\sum_{j k} \frac{\delta \bar{\sigma}_{x g}}{\delta p_{j}}\left\langle\delta p_{j} \delta p_{k}\right\rangle \frac{\delta \bar{\sigma}_{x g^{\prime}}}{\delta p_{k}}
$$

The covariance of $\bar{\sigma}_{x g}$, the flux-weighted group cross section for reaction $x$, is a function of the derivative of the cross sections with respect to the parameters $p_{j}$ and $p_{k}$, that is, the cross section sensitivity to the RP, and the RPCM given as $\left\langle\delta p_{j} \delta p_{k}\right\rangle$ which are quantities stored in FILE32. On the other hand, if the CSCM listed in FILE33 is $M(\sigma)$, the $\left\langle\delta \bar{\sigma}_{x g} \delta \bar{\sigma}_{x g^{\prime}}\right\rangle$ is obtained as

$$
\left\langle\delta \bar{\sigma}_{x g} \delta \bar{\sigma}_{x g^{\prime}}\right\rangle=\frac{1}{\varphi_{g} \varphi_{g^{\prime}}} \int_{E_{g}}^{E_{g+1}} \int_{E_{g^{\prime}}}^{E_{g^{\prime}+1}} \varphi(E) \varphi\left(E^{\prime}\right) M(\sigma) d E d E^{\prime}
$$

where

$$
\varphi_{g}=\int_{E_{g}}^{E_{g+1}} \varphi(E) d E
$$


Table 1. Cross section at thermal energy $(0.0253 \mathrm{eV})$ and capture resonance integral.

\begin{tabular}{lll}
\hline Quantity & ENDF $/$ B-VII.0 & New ${ }^{48}$ Ti evaluation \\
\hline$\sigma_{\gamma}$ & 7.84 & $8.32 \pm 0.23$ \\
$\sigma_{s}$ & 4.36 & $4.04 \pm 0.23$ \\
$\sigma_{t}$ & 12.20 & $12.35 \pm 0.23$ \\
$I_{\gamma}$ & 3.68 & $3.78 \pm 0.23$ \\
\hline
\end{tabular}

Table 2. Computer storage size for covariance libraries.

\begin{tabular}{lr}
\hline Library & \multicolumn{1}{c}{ Size } \\
\hline RPCM_LIB (resonance parameter covariance) & $904 \mathrm{~KB}$ \\
CSM44_LIB (44-group covariance) & $95 \mathrm{~KB}$ \\
CSCM_380LIB (380-group covariance) & $72 \mathrm{MB}$ \\
\hline
\end{tabular}

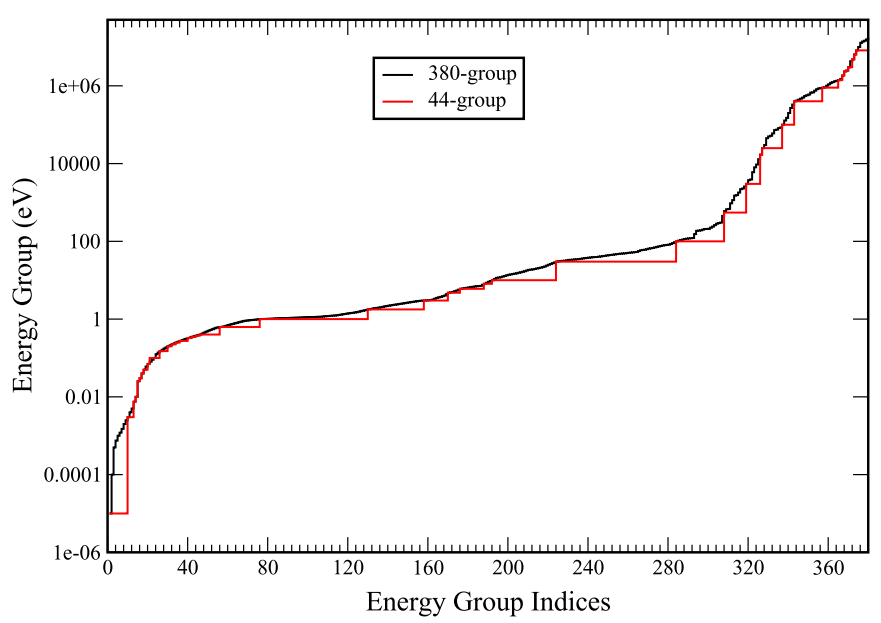

Fig. 3. Comparison of the 380 group (black line) and 44 group (red line) structures.

Here $\varphi_{g}$ is the energy-dependent neutron flux in the energy group $g$. The results of equations (1) and (2) are equivalent as long as the conversion of $\left\langle\delta p_{j} \delta p_{k}\right\rangle$ into $M(\sigma)$ is properly done [5]. The FILE33 CSCM representation is intended to characterize the variances of the cross sections within a specified energy region, and the correlation between cross sections of adjacent energy regions. The choice of CSCM over the RPCM is expected to lead to a reduction in computer storage. However, a gain in computer use, meaning data storage and perhaps computer time, may not preserve the accuracy sought in practical applications such as, for instance, the establishing of criticality safety limits and their related uncertainties. The issues, pros and cons, in connection with the conversion from FILE32 to FILE33 representation is investigated in the following sections.

\subsection{File32 to File33 Conversion for ${ }^{48} \mathrm{Ti}$}

The conversion from the RPCM to the CSCM representation has been investigated by means of using two energy group configurations, a coarser and a finer energy group structures.

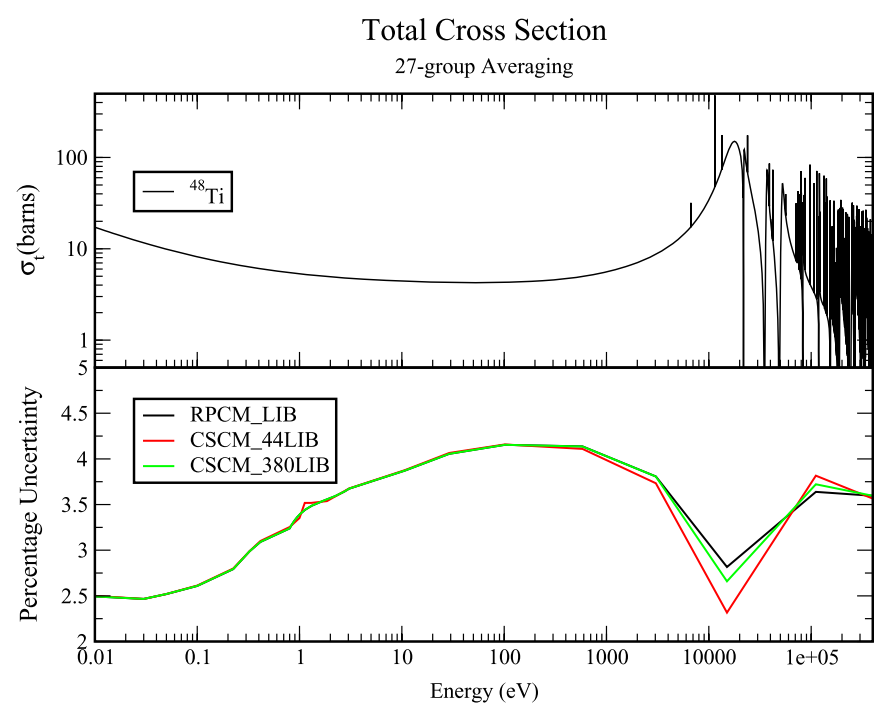

Fig. 4. 27-group calculated covariance for the total cross section.

The finer group structure consists of an energy grid of 381 boundaries, i.e. 380 energy groups, whereas the coarse energy group is rather composed of 44-energy groups chosen as a subset of the 380-group structure. A graphically representation of these group structures is displayed in Figure 3. While the 380-group representation is very detailed approaching to a single line in the picture (black line) the 44-group (red line) clearly shows the effect of the coarse energy mesh. The conversions were carried out with the AMPX code system [6] using the modules, PRELL, PRILOSEC, PUFF, and COVCONV. The detailed information on how to use these AMPX modules can be found in the AMPX manual. A constant energy-dependent neutron flux, required for weighing the covariance as indicated in equations (2) and (3), were used in the conversion. It should be recalled that the results presented here are for the energy region $10^{-5} \mathrm{eV}-$ $400 \mathrm{keV}$ corresponding to the resolved resonance evaluation of ${ }^{48} \mathrm{Ti}$. For the 44-group structure 35-energy groups fall under $400 \mathrm{keV}$ whereas 343-energy groups have energies under $400 \mathrm{keV}$ for the 380 -group structure. The exercise led, respectively, to two ENDF formatted cross-section libraries including the 44-group and the 380-group covariance generated in the conversion of the RPCM to the CSCM representation. The data libraries used in the studies presented in this work are identified as follows: a) the covariance library based solely on the resonance parameter representation will be referred to as the RPCM LIB; $b$ ) the covariance library resulting from the conversion from the RPCM to the CSCM using the 44-group structure is referred to as the CSCM_44LIB; c) lastly, the 380-group library is 


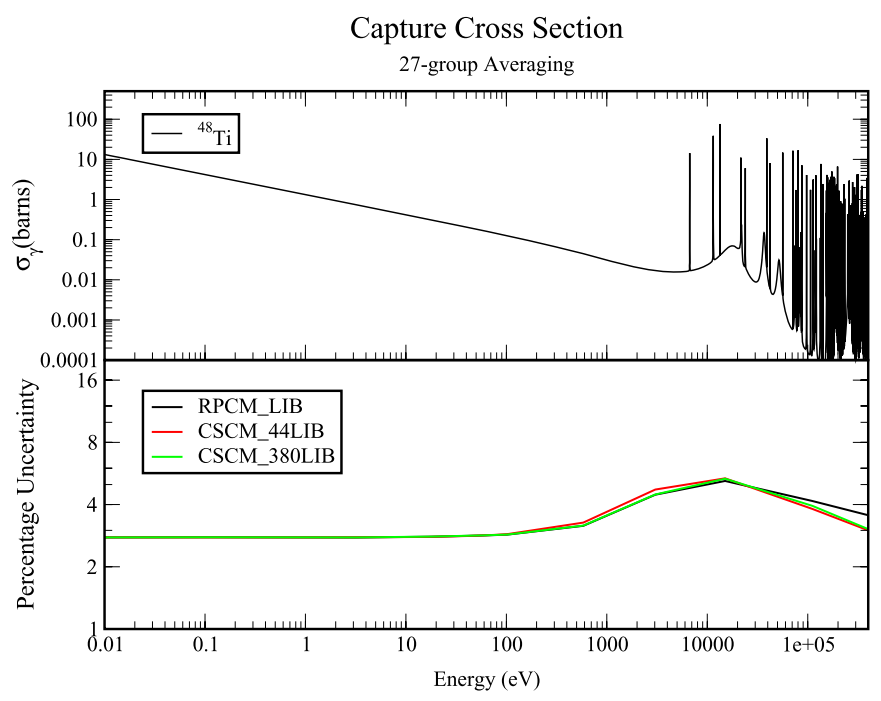

Fig. 5. 27-group calculated covariance for the capture cross section.

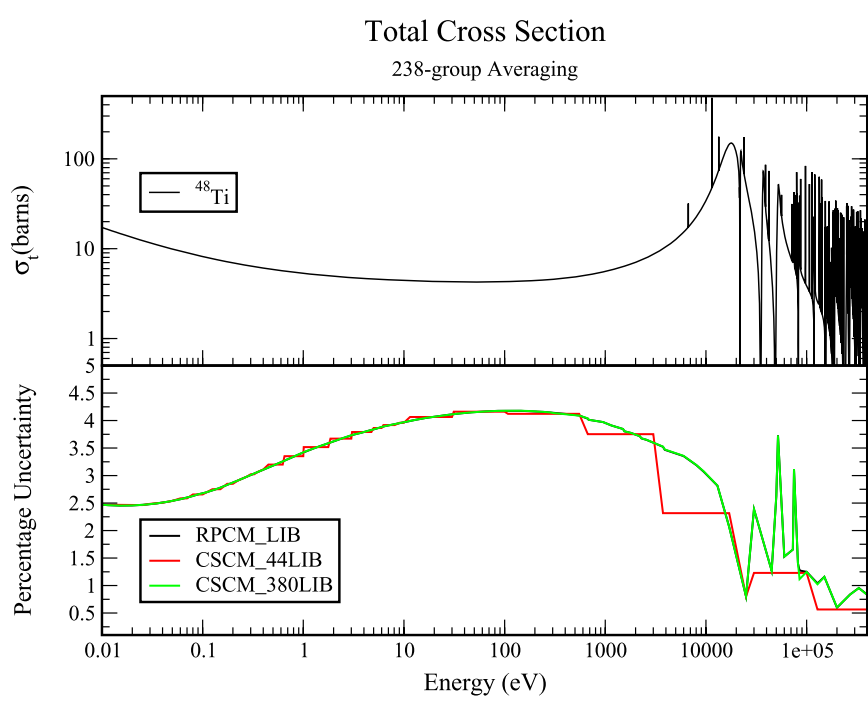

Fig. 6. 238-group calculated covariance for the total cross section.

named CSCM_380LIB. For the sake of information the computer storage size of the covariance portion of these libraries are listed in Table 2.

\subsection{Tests and results}

For practical applications the covariance information in the nuclear data libraries such as ENDF, JEFF, JENDL and others must be processed in a form suitable for use in sensitivity uncertainty (S/U) analysis. There are several tools that can make use of the processed libraries for error propagation studies. The most common processed form of the covariance data these codes use can take either the COVERX [7] or the BOXER format. The S/U code may have been built to accept both forms. In the study carried out here both forms of the processed covariance were generated.

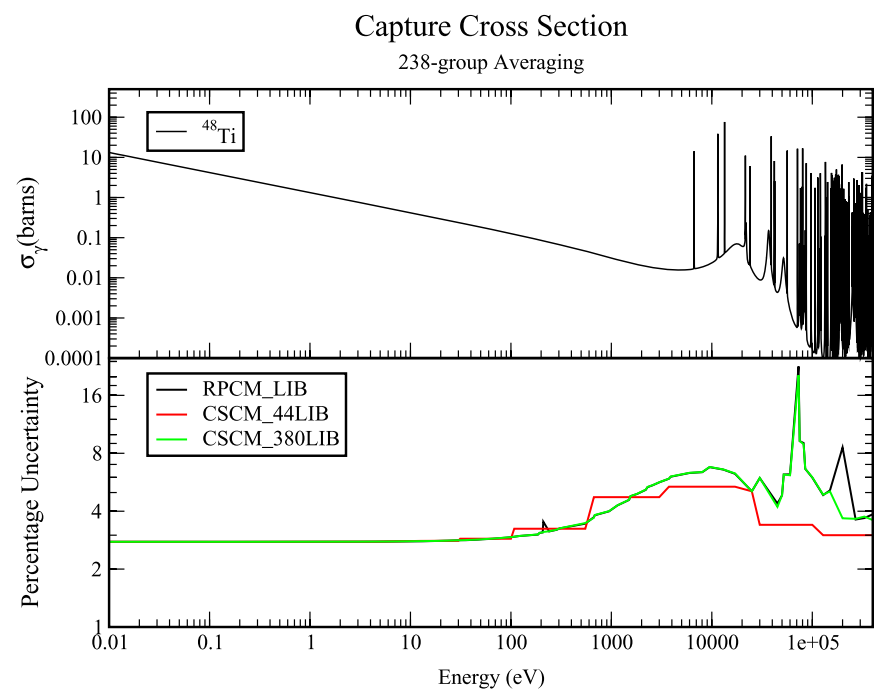

Fig. 7. 238-group calculated covariance for the capture cross section.

The impact of the covariance representation in the resonance region of the ${ }^{48} \mathrm{Ti}$ evaluation was assessed by processing the libraries RPCM LIB, CSCM 44LIB, and CSCM 380LIB on the basis of two energy group structures. The energy groups chosen are those listed in the SCALE code system, [6] namely the 27-group and the 238 -group. The results were obtained by processing the covariance information in the libraries using the ERRORR module of the NJOY code system [8] with a constant energy-dependent neutron flux. The reported results are for the ${ }^{48} \mathrm{Ti}$ total and capture cross sections. The results are displayed in the graphical form. For each group structure (27-group and 238-group) three calculations were carried out corresponding to the three libraries. The results are overlaid for a better visualization of the impact of using the three covariance representations. The results for the total and capture cross sections are shown in Figures 4 and 5, respectively. The upper portion of each picture represents the corresponding cross section whereas the bottom part is the calculated uncertainty. It appears that for the 27-group generated covariance the CSCM representation underestimate the uncertainty compared to the RPCM for the total cross section in the energy range where resonances exist. The same trend is also observed for the capture cross section but nevertheless the difference where the resonances start are very small. The results based on the 238goup are shown in Figures 6 and 7 , respectively. The two results for the total and the capture cross sections indicate that in the energy region where no resonance is present apparently the 44-group covariance representation seems to be represented reasonably well. However, as the resonances arise the results based on the 27-group representation deteriorates showing large differences in comparison with the RPCM calculations. On the other hand the CSCM based on the 380-group is in very good agreement with the RPCM. Nonetheless the improvement on the results based on the 380-group are based on a covariance matrix that requires a large computer storage size as indicated in Table 2 and therefore not 
offering any advantage over the resonance parameter representation. The results of these calculations lead to some inferences:

- care must be taken when using few-group covariance representations. It may be appropriate for the energy range where the data are smooth and the resonances are not present as for instance in the high energy region;

- for a detailed covariance results the use of the RPCM is recommended;

- conversion of the RPCM to CSCM using a fine energygroup structure must be examined so as to assure that the computer allocation size is not overwhelming larger than that of the RPCM;

- in the resonance region few-group representation is acceptable when a general overview of the impact of covariance results is sought.

\section{Conclusions}

This paper presents, at a certain extent, a discussion on the representation of the covariance data in the evaluated nuclear data libraries. The resonance parameter covariance for ${ }^{48} \mathrm{Ti}$ was used in the study. The impact of using the RPCM as opposed to the CSCM representation and the results of calculations based on these covariance representations are presented. Three covariance representations were used in the study, namely, one based on the resonance parameters, a coarser group covariance using 44-group and a finer group covariance using 380-energy groups. The results suggested that the use of the RPCM is preferred over the CSCM representation. However there are situation in which a fine-group group structure may be advised as for instance in the case where the RPCM lends itself to a huge covariance matrix representation. Another alternative could be to reduce the size of the RPCM by using a compact representation of the covariance or another preferred approach.

There are other issues that were not addressed in this paper such as the impact of the weighting spectrum in the covariance conversion from RPCM to CSCM. While this issue may impact the results, the conversion of the resonance parameter representation to cross section representation of the covariance together with the choice of the energy group structure is believed to be a more dominant subject. However, work is underway to understand the impact of the covariance weighting in the conversion process. Other subjects under examination are the impact of the cross section energy self-shielding and the temperature effect in the covariance. The results presented in this work were obtained for room temperature and infinite diluted cross sections. Also, no attempt has been made in this paper to examine the implication of the RPCM and CSCM representations in practical applications such as the magnitude of the propagated uncertainty to $k_{e f f}$ for system sensitive to the ${ }^{48} \mathrm{Ti}$.

\section{References}

1. N.M. Larson, Updated Users Guide for SAMMY: Multi-Level R-Matrix Fits to Neutron Data Using Bayes' Equations, (ENDF-364/R2, Oak Ridge National Laboratory, 2008), available at the Radiation Safety Information Computational Center (RSICC) as PSR-158

2. L. Leal, K. Guber, G. Arbanas, D. Wiarda, P. Koehler, A. Kahler, Resonance evaluation of Titanium-48 including covariance for criticality safety applications, in International Conference on Nuclear Criticality Safety, Edinburgh, United Kingdom, 2011

3. K.H. Guber, P.E. Koehler, D. Wiarda, J.A. Harvey, Neutron Cross-Section Measurements on Structural Materials at ORELA, in Proceedings of the International Conference on Nuclear Data for Science and Technology, Jeju Island, Korea, 2010

4. ENDF-6 Formats Manual, Formats and Procedures for the Evaluated Nuclear Data Files ENDF/B-VI and ENDF/ $B$-VII, edited by M. Herman, A. Trkov, Report-BNL90365-2009 Rev. 1 (National Nuclear Data Center, Upton, NY, 2010)

5. L. Leal, D. Mueller, G. Arbanas, D. Wiarda, H. Derrien, Impact of the ${ }^{235} \mathrm{U}$ Covariance Data in Benchmark Calculations, in International Conference on the Physics of Reactors Nuclear Power: a sustainable resource CasinoKursaal Conference Center, Interlaken, Switzerland, 2008

6. B.T. Rearden, M.A. Jesse, et al., SCALE Code System, ORNL/TM-2005/39 Version 6.2.1, 2016

7. J.D. Drischler, The COVERX Service Module of the FORSS System, ORNL/TM-7181, Oak Ridge National Laboratory, Oak Ridge, Tenn, 1980

8. R.E. MacFarlane, D.W. Muir, A.C. Kahler, The NJOY Nuclear Data Processing System, Version 2012, LA-UR-1227079, Los Alamos National Laboratory, 2012 